\title{
Clinical utility of raltegravir for the treatment of HIV infection in children and adolescents
}

This article was published in the following Dove Press journal:

Pediatric Health, Medicine and Therapeutics

8 August 2013

Number of times this article has been viewed

\author{
James Nuttall' \\ Tammy Meyers ${ }^{2}$ \\ Brian Eley' \\ 'Paediatric Infectious Diseases \\ Unit, Red Cross War Memorial \\ Children's Hospital and Department \\ of Paediatrics and Child Health, \\ University of Cape Town, Cape \\ Town, South Africa; ${ }^{2}$ Department \\ of Paediatrics, Chris Hani \\ Baragwanath Hospital and Faculty \\ of Health Sciences, University of \\ the Witwatersrand, Johannesburg, \\ South Africa
}

\begin{abstract}
Raltegravir (RAL) is the first integrase strand transfer inhibitor and has been shown to provide potent antiretroviral (ARV) activity against human immunodeficiency virus type 1 (HIV-1) in both ARV treatment-naïve and treatment-experienced individuals. Following initial US Food and Drug Administration (FDA) approval of RAL for treatment of HIV-1-infected adults in 2007, an ongoing pharmacokinetic, safety, and efficacy study in ARV-experienced children and adolescents led to extension of FDA approval to children and adolescents aged 2-18 years in 2011. Availability of chewable tablets for children aged 2-11 years is a significant advantage, and twice-daily dosing is recommended based on pharmacokinetic parameters. Granules for oral suspension in children 4 weeks to 2 years of age are currently under evaluation and clinical trials in neonates are imminent. Coadministration of RAL and the anti-tuberculosis drug rifampin (RIF) results in reduced RAL exposure. Evaluation of a double RAL dosing strategy in children requiring cotreatment with RIF is planned. RAL is generally well tolerated and has a good overall safety profile. Further data is required for children before RAL can be recommended in first-line ARV treatment regimens. RAL is also under investigation for use in preventing mother-to-child transmission both during pregnancy and in the HIV-exposed neonate. Currently, the main therapeutic role for RAL in children is for treatment failure and multi-drug resistant cases where the inclusion of RAL in combination with optimal background therapy has demonstrated successful outcomes. Increased availability of RAL and the introduction of second-generation integrase inhibitors are likely to further extend the utility of this class of ARV drugs.
\end{abstract}

Keywords: antiretroviral therapy, integrase strand transfer inhibitor, raltegravir, HIV-1

\section{Introduction}

Despite widespread implementation of programs for the prevention of mother-to-child transmission (PMTCT) of human immunodeficiency virus (HIV), many children, and particularly those in resource-limited settings, continue to acquire HIV infection during pregnancy, birth, or breastfeeding. For children able to access antiretroviral therapy (ART), HIV infection has changed from a fatal disease to a chronic manageable medical condition. Increased survival brings with it the need for lifelong treatment and a focus on quality of life. Tolerability, toxicity, and durability of ART regimens are critical factors to be considered. Older children and adolescents may harbor extensive antiretroviral (ARV) drug resistance as a result of prior treatment with suboptimal regimens and adherence difficulties. These patients require new generations of ARV drugs including novel drug classes in order to remain healthy. The development of integrase inhibitors, a new class of ARV drugs, and the recent approval of raltegravir 
(RAL) (Isentress; Merck and Co, Inc, Whitehouse Station, NJ, USA) for use in children and adolescents requires a critical appraisal of current pediatric ARV regimens in order to consider the optimal role that this new class of drugs, and RAL in particular, should play. This review focuses on the current state of knowledge and experience with RAL, with particular attention to its role in HIV-infected children and adolescents.

Almost all infants and young children acquire HIV infection as a result of vertical transmission from their mothers. Depending on the timing of HIV infection (during early pregnancy, around birth, or during the postnatal period via breastmilk) and other factors (eg, nutritional state and concomitant infections), untreated HIV infection may rapidly progress to a life-threatening (and often fatal) disease; alternatively, it may instead remain more stable for months and up to a few years with later onset of disease manifestations. Initiation of ART before 3 months of age regardless of clinical stage of disease or CD4 (cluster of differentiation 4) count has been shown to reduce early infant mortality and disease progression by $75 \%$, as compared to deferred treatment. ${ }^{1}$

The goals of ART in children include reducing HIVrelated mortality and morbidity, preserving or restoring immune function, maximal and durable suppression of viral replication, preventing the emergence of viral drug resistant mutations, minimizing drug-related toxicity, maintaining normal physical growth and neurocognitive development, improving quality of life, and reducing the risk of sexual transmission to discordant partners in adolescents who are sexually active. ${ }^{2}$ Early ARV treatment approaches using mono- or dual-drug regimens resulted in treatment failures with viral non-suppression or rebound, and the development of viral resistance after only a few months or years of treatment. Current state-of-the-art initial ART for children is based on combination regimens incorporating at least three drugs from at least two ARV drug classes. A dual nucleoside/ nucleotide reverse transcriptase inhibitor (NRTI) backbone combined with either a non-nucleoside reverse transcriptase inhibitor (NNRTI) or protease inhibitor (PI) is recommended for children. ${ }^{2}$ Compared to earlier treatment approaches, these regimens have shown greater durability and resulted in significant reductions in the mortality and morbidity of HIV-infected children. Fewer opportunistic infections and hospitalization episodes, and improved growth and neurocognitive function, have been reported during the period since highly-active ART was introduced..$^{3-6}$

However, despite the successes of long-term ART, issues of drug toxicity, drug-drug interactions, problems with adherence to treatment, and drug resistance remain challenging and may result in limitation of treatment options. In children, resistance to ARV drugs within or across drug classes may be acquired as a result of suboptimal adherence to treatment, prior exposure to regimens with reduced potency including non-suppressive single drug regimens used in PMTCT programs, or by mother-to-child transmission of drug-resistant virus. ${ }^{7,8}$ Adverse effects of ARV drugs are generally less common in children compared to adults but include hypersensitivity reactions, mitochondrial toxicity, hepatotoxicity, gastrointestinal disturbance, bone marrow suppression, and dyslipidemia.9,10 Careful drug selection and sequencing are needed in order to preserve future treatment options; similarly, close follow-up of the child and caregiver are essential in assessing adherence to prescribed treatment, tolerance of medication, and response to therapy. ${ }^{2}$ Important additional factors that impact the initiation and adjustment of ART in children are summarized in Table 1.

Guidelines for treatment of HIV-infected children are evolving as new data become available. Although prospective, randomized, controlled clinical trials offer the best evidence for formulation of guidelines, most ARV drugs are approved for use in pediatric patients based on efficacy data from clinical trials in adults. Such trials frequently make use of surrogate markers of efficacy (HIV-1 ribonucleic acid [RNA] and CD4+ cell count) rather than clinical end points,

Table I Important factors to consider when initiating or adjusting antiretroviral therapy in children

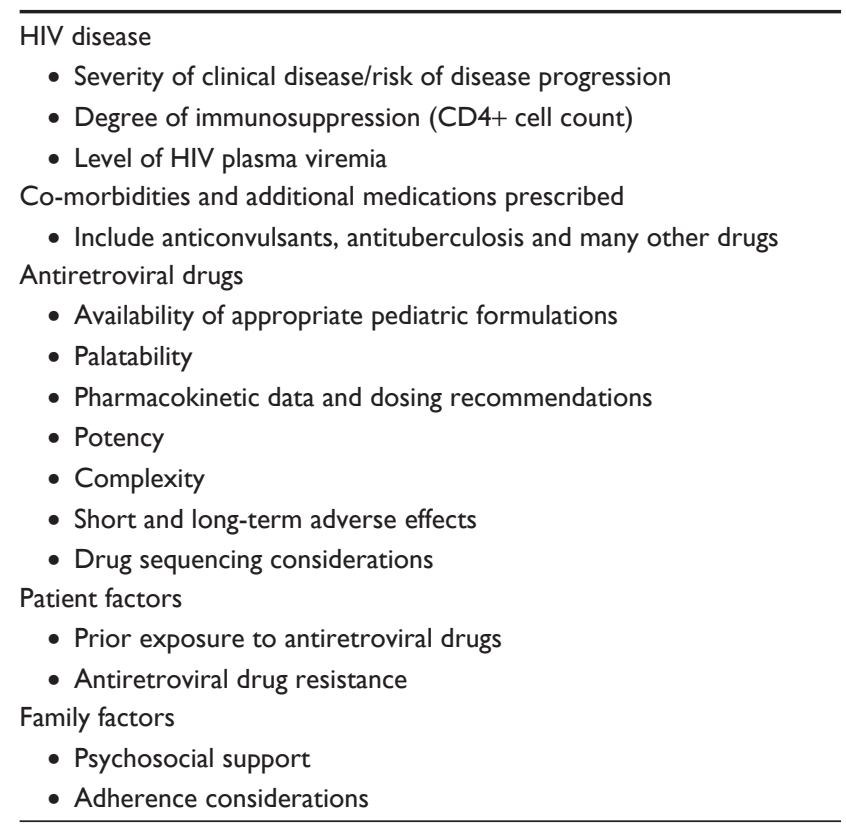

Abbreviation: HIV, human immunodeficiency virus. 
together with supporting pharmacokinetic (PK) and safety data from Phase I/II trials in children. ${ }^{2}$

RAL, the first integrase strand transfer inhibitor (ISTI), provides potent ARV activity against HIV-1 in both ARVnaïve and ARV-experienced patients. In October 2007, the US Food and Drug Administration (FDA) approved RAL for salvage therapy in ARV-experienced adults, and in 2009 approval was extended to first-line treatment of ARV-naïve adults. In December 2011, approval was extended to children and adolescents aged $2-18$ years. ${ }^{11}$

\section{Pharmacology of RAL Mechanism of action}

The HIV-1 integrase enzyme is responsible for the transfer of viral DNA (deoxyribonucleic acid) strands into the host genome, an essential step in the replication cycle of the virus. Viral DNA transfer involves several key steps. Initially, integrase combines with viral DNA and host cofactors to form the preintegration complex. This is followed by $3^{\prime}$ processing within the cytoplasm of the CD4 cell, during which integrase catalyzes the cleavage of a dinucleotide from both $3^{\prime}$ ends of the viral DNA. The preintegration complex then crosses the nuclear membrane to enter the nucleus, where integrase cleaves host DNA and inserts the cleaved viral DNA 3' ends into opposing strands of host cell chromosomal DNA. HIV DNA integration may occur at many different sites within the host genome, particularly within active transcription units. The host cell lens epithelium-derived growth factor (LEDGF/p75), an integral component of the preintegration complex, improves the efficiency of integration and mediates targeting of active transcription units. After strand transfer has been completed, host cell enzymes repair the single-strand gaps and $5^{\prime}$ overlapping segments to establish a stable provirus. ${ }^{12-15}$

RAL binds to the catalytic site of integrase only when the enzyme is complexed to viral DNA, thus preventing the preintegration complex from binding to host cell DNA and the transfer of viral DNA to the host genome. ${ }^{14,16}$ It exhibits potent in vitro activity against HIV-1 in human T-cell culture. The geometric mean $95 \%$ inhibitory concentration $\left(\mathrm{IC}_{95}\right)$ is $33 \mathrm{nmol} / \mathrm{L}$ (15 ng/mL) in 50\% human serum. ${ }^{17,18}$

\section{PK profile}

Most of the PK characteristics of RAL have been established during the study of adult subjects. The drug is rapidly absorbed from the gastrointestinal system, reaching a maximum concentration $\left(\mathrm{T}_{\max }\right)$ approximately 3 hours after oral administration. At therapeutic dose, the elimination half-life of RAL varies between 7-12 hours. PK steady state is achieved within 2 days of initiating therapy. The drug is $83 \%$ bound to human plasma protein. The distribution of the drug is mainly to plasma, the gastrointestinal tract, and excretory organs. In animal studies the volume of distribution ranges from $0.2-2 \mathrm{~L} / \mathrm{kg}$. At the adult dose of $400 \mathrm{mg}$ twice-daily the mean plasma trough concentration $\left(\mathrm{C}_{\text {trough }}\right)$ exceeds the mean $\mathrm{IC}_{95}{ }^{17-21}$

Based on efficacy trials, ${ }^{22,23,24}$ RAL dosed at $400 \mathrm{mg}$ twicedaily was licensed for use in adult patients. A recent noninferiority trial that compared $800 \mathrm{mg}$ once-daily to $400 \mathrm{mg}$ twice-daily RAL dosing in combination with two NRTIs in untreated HIV-infected adults showed that once-daily dosing was inferior and thus could not be recommended in routine clinical practice. ${ }^{22} \mathrm{PK}$ comparison of once-daily versus twicedaily dosing showed similar area under the concentrationtime curve from 0-24 hours $\left(\mathrm{AUC}_{0-24}\right)$, but a six-fold lower plasma $\mathrm{C}_{\text {trough }}$ in those who received once-daily RAL. ${ }^{25}$

For an ARV drug to inhibit HIV-1 replication in the central nervous system (CNS) it should cross the blood-brain barrier and achieve a sufficiently high cerebrospinal fluid (CSF) concentration. A study evaluated RAL concentration in 25 pairs of plasma and CSF specimens from 16 adult patients being treated with RAL-containing regimens. All plasma RAL concentrations were above the $\mathrm{IC}_{95}$, the median being $448 \mathrm{ng} / \mathrm{mL}$. RAL was detected in 24 of the CSF specimens. The median RAL concentration in the CSF specimens was $18.4 \mathrm{ng} / \mathrm{mL}$, and 13 had concentrations above the $\mathrm{IC}_{95}$. The CSF:plasma ratios were low, ranging from $0.01-0.61$, with the median ratio being $0.03 .{ }^{26}$ Another study evaluated 25 specimen pairs from 18 patients. RAL was detected in all CSF specimens, with a median concentration of $14.5 \mathrm{ng} / \mathrm{mL}$. The median plasma:CSF ratio was 0.06. HIV RNA was detectable ( $>50$ copies $/ \mathrm{mL}$ ) in $38 \%$ of plasma and $4 \%$ of CSF specimens. Because of the small sample size it was not possible to determine factors associated with a detectable CSF HIV RNA. ${ }^{27}$ Therefore, the clinical implications of these observations remain to be determined. The first case report was recently published looking at integrase inhibitor resistance in the CNS in the absence of plasma resistance, in an adult receiving once-daily RAL. To date there have not been similar reports in patients receiving twice-daily RAL. ${ }^{28}$

RAL demonstrates marked intra- and interpatient PK variability. In a study of 86 patients, the interpatient variability as measured by the plasma $\mathrm{C}_{\text {trough }}$ coefficient of variation was $110 \%$. In 13 of these patients who each had three or more specimens analyzed, the median intrapatient coefficient of variation was $128 \%$; in seven patients in whom ten or more 
specimens were analyzed, median intrapatient coefficient of variation was $245 \%{ }^{29}$ In another study of 15 patients, marked variability of the $\mathrm{AUC}_{0-4}$ and $\mathrm{AUC}_{0-12}$ was documented. Furthermore, intrapatient variability accounted for a large proportion of overall variability. ${ }^{30}$ RAL variability limits the usefulness of therapeutic drug monitoring during ART. Concern about the potential effect of PK variability on longterm RAL effectiveness has also been expressed. ${ }^{30}$ A comparative study showed that patients who swallowed RAL after first chewing the tablets experienced significantly higher absorption and lower interpatient PK variability than those who swallowed tablets intact. In vitro experiments confirmed poor dissolution of whole tablets relative to crushed tablets. In particular, less than $10 \%$ of intact tablets tested in an acidic medium dissolved within 2 hours. These results suggest that improved oral formulations should result in improved drug exposure. ${ }^{31}$ Prior food intake may also affect the absorption of RAL. Relative to patients who were administered RAL during a fasting state, a low-fat meal resulted in moderate reduction in absorption but had no effect on $\mathrm{C}_{\text {trough }}$, whereas a high-fat meal increased absorption and $\mathrm{C}_{\text {trough }}$. A moderatefat meal produced a similar PK profile to the fasting state. Despite these PK differences, no safety concerns were identified, and RAL may be given without regard for prior food ingestion. ${ }^{32}$

RAL is metabolized by hepatic glucuronidation via uridine diphosphate glucuronosyltransferase (UGT) isoenzyme 1A1 to RAL glucuronide. UGT isoenzymes $1 \mathrm{~A} 3$ and 1A9 play lesser roles in the glucuronidation of RAL; ${ }^{31}$ the drug is rapidly eliminated from the body in either urine or feces. After administration of oral $\left[{ }^{14} \mathrm{C}\right] \mathrm{RAL}$ to eight adult volunteers, $31.8 \%$ of the drug was recovered in urine and $51.2 \%$ in feces. Two chromatographic peaks were identified in urine, $22.9 \%$ of the total oral dose was RAL glucuronide, and $8.8 \%$ was the unchanged parent compound. Only one peak due to the parent compound was observed in feces. No evidence of enterohepatic circulation was observed. Analysis after intravenous $\left[{ }^{14} \mathrm{C}\right] \mathrm{RAL}$ administration to rats showed that $85 \%$ of the drug that was secreted into bile was RAL glucuronide, while $100 \%$ of fecal radioactivity was caused by the parent compound. This suggests that following secretion into bile, RAL glucuronide is hydrolyzed in feces to the parent compound, resulting in the observed single chromatographic peak. ${ }^{18,33}$ Decreased UGT 1 A1 activity occurs in subjects with the $* 28 / * 28$ genotype. Although the $\mathrm{AUC}_{0-\infty}$ and maximum plasma concentration of RAL were moderately increased in subjects with this genotype, these PK alternations are not considered clinically significant. ${ }^{34}$
Furthermore, studies have shown that dose adjustments are not necessary in patients with hepatic insufficiency, including those with end-stage liver disease, nor in those with severe renal insufficiency. ${ }^{35,36}$

\section{PKs and dosing in children}

Recent evidence from clinical trials has provided the necessary data required for licensing RAL for use in pediatric populations. The ongoing International Maternal Pediatric Adolescent AIDS Clinical Trials (IMPAACT) P1066 study is a prospective, non-randomized open-label dose-finding trial of RAL plus optimal background therapy (OBT) in ARV treatment-experienced children and adolescents. ${ }^{37-40,41-45}$ The study comprises 6 cohorts (I: $12-18$ years; IIA and IIB: 6-11 years; III: 2-5 years; IV: 6 months-2 years; V: 4 weeks-6 months) and evaluates the PK profile, safety, tolerability, and efficacy of three RAL formulations: the adult tablet (400 mg), pediatric chewable tablets (100 mg and $25 \mathrm{mg}$ ) and granules for suspension (Table 2).

PK evaluation of fasting adolescents aged 12-18 years showed that at a dose of $400 \mathrm{mg}$ twice-daily, the use of the film-coated poloxamer formulation that is registered for use in adults resulted in adequate drug exposure comparable to the PK profile generated in adult patients. ${ }^{37,38}$

Two formulations were evaluated amongst children aged 6-11 years, film-coated poloxamer tablets and chewable ethylcellulose tablets, both administered initially at a dose

Table 2 Overview of IMPAACT (International Maternal Pediatric Adolescent AIDS Clinical Trials) PI066 cohorts and dosing in treatment-experienced children and adolescents administered raltegravir plus an optimized background regimen

\begin{tabular}{ll}
\hline Cohort description & Results \\
\hline Cohort I: adolescents & Raltegravir, film-coated poloxamer tablets \\
aged I2-I8 years & $400 \mathrm{mg}$ twice daily provided adequate \\
pharmacokinetic exposure & Raltegravir, film-coated poloxamer tablets \\
Cohort IIA: children & 400 mg twice daily provided adequate \\
aged 6-II years & pharmacokinetic exposure \\
Cohort IIB: children & Raltegravir ethylcellulose chewable tablets, \\
aged 6-II years & $\begin{array}{l}\text { daily provided adequate pharmacokinetic } \\
\text { exposure }\end{array}$ \\
Cohort III: children & Raltegravir ethylcellulose chewable tablets, \\
aged 2-5 years & 6 mg/kg (maximum dose 300 mg) twice \\
& daily provided adequate pharmacokinetic \\
& $\begin{array}{l}\text { exposure } \\
\text { Raltegravir oral granules, } 6 \text { mg/kg twice } \\
\text { daily provided adequate pharmacokinetic } \\
\text { exposure }\end{array}$ \\
aged 6-23 months & Pharmacokinetic, dose finding, safety and \\
Cohort V: children aged & efficacy evaluation underway \\
\hline
\end{tabular}


of $8 \mathrm{mg} / \mathrm{kg}$ twice-daily. ${ }^{39,40}$ Prior single-dose evaluation of the chewable tablets in healthy adults had shown that, in comparison to the film-coated tablet, a similar $\mathrm{C}_{\text {trough }}$, higher $\mathrm{AUC}_{0-\infty}$ and earlier $\mathrm{T}_{\max }$ were achieved with an identical milligram dose of the chewable tablets. ${ }^{46}$ The studies in children aged 6-11 years demonstrated that both formulations at the prescribed dose achieved adequate drug exposure..$^{39,40}$ The chewable tablet was associated with less PK variability and lower oral clearance. Differences in clearance suggested greater relative bioavailability of the chewable tablet. Based on the pharmacological profile, the dose of the chewable tablet was lowered to $6 \mathrm{mg} / \mathrm{kg}$ twice-daily in this age group. ${ }^{40}$ The chewable tablet has also been successfully evaluated in children aged 2-5 years at a dose of approximately $6 \mathrm{mg} / \mathrm{kg}$ twice-daily, to a maximum of $300 \mathrm{mg}$ twice-daily. A similar PK profile was obtained to that observed in children aged 6-11 years. ${ }^{41}$

For children aged 6-23 months an oral granule formulation was evaluated. ${ }^{42} \mathrm{~A}$ prior study in healthy adult volunteers showed that in comparison to film-coated poloxamer tablets, the oral granule formulation achieved an earlier $\mathrm{T}_{\text {max, }}$, moderately higher $\mathrm{AUC}_{0-\infty}$, and similar $\mathrm{C}_{\text {trough }}$ measurements. ${ }^{46} \mathrm{At} \mathrm{a}$ dose of approximately $6 \mathrm{mg} / \mathrm{kg}$ twice-daily, similar PK values were achieved to those observed in 2-11-year-old children receiving the chewable tablet formulation..$^{42}$ An ongoing objective of the IMPAACT P1066 trial is the evaluation of the oral granule formulation of RAL in children aged 4 weeks to 5 months.

Because RAL is currently being used in HIV-infected pregnant women, and in future may be considered in neonates for PMTCT or treatment of HIV infection, PK evaluation of the drug in newborn infants is underway. An initial report described three newborns exposed to maternal therapeutic RAL during late pregnancy. In two newborns, RAL concentrations measured within 3 hours post-delivery were 7 and 9.5 times higher than paired maternal specimens. ${ }^{47}$ Another study evaluated three preterm newborns exposed to maternal RAL administered antenatally for PMTCT. Shortly after birth, all three had therapeutic RAL plasma concentrations ( $>15 \mathrm{ng} / \mathrm{mL}$ ), including two whose mothers had been initiated on RAL 22.5 and 14 hours before delivery. RAL remained above therapeutic concentration for up to 5 days after delivery. ${ }^{48}$ The IMPAACT P1097 trial examined the washout PK of transplacentally-acquired RAL in term newborns during the first 36 hours after birth. Median cord blood concentration was $957 \mathrm{ng} / \mathrm{mL}, 1.48$ times higher than the median maternal RAL concentration at delivery. RAL concentration remained above the $\mathrm{IC}_{95}$ throughout the sampling period in all but one of the newborns evaluated. Median terminal half-life of RAL was 26.6 hours. ${ }^{49}$ Neonatal dosing regimens, safety, and PK considerations will be addressed in the forthcoming IMPAACT P1110 trial.

\section{Drug interactions \\ Rifamycins}

Rifampin (RIF) induces several enzymes, including glucuronosyltransferases that are responsible for metabolizing RAL; coadministration results in significantly lower RAL PK exposure. A study evaluated the effect of $600 \mathrm{mg}$ of RIF once-daily on the PK of either a single $400 \mathrm{mg}$ dose of RAL (group 1) or a single $800 \mathrm{mg}$ dose of RAL (group 2) in healthy adults. Subjects in group 1 experienced significantly lower drug exposure relative to controls. Patients in group 2 experienced a $53 \%$ decrease in mean $\mathrm{C}_{\text {trough }}$, but higher $\mathrm{AUC}_{0-12}$ and maximum concentration $\left(\mathrm{C}_{\max }\right)$ compared to a control group that received RAL $400 \mathrm{mg}$ twice-daily but no RIF. These results suggested that doubling the dose of RAL could partially compensate for the effect of RIF on RAL metabolism, but that the clinical effectiveness of this strategy should be evaluated..$^{50}$ Based on these data, the US FDA approval was updated in February 2009 to include the recommendation to increase the RAL dose to $800 \mathrm{mg}$ twice-daily during coadministration with RIF. Furthermore, the IMPAACT P1101 study will shortly commence patient enrollment. It aims to evaluate the safety and effective dosing of RAL-containing ART in children receiving anti-tuberculosis (TB) medication. Double dosing of the chewable ethylcellulose RAL formulation in combination with other ARVs will be evaluated in children (aged 3-12 years) coinfected with TB and taking a RIF-containing anti-TB regimen.

The efficacy of three ARV regimens was recently evaluated in a Phase II randomized-control clinical trial of HIVinfected, ARV-naïve adults with TB that were cotreated with RIF-containing anti-TB medication. One hundred and fiftythree randomized subjects were included in the analysis. All of these subjects received a standard NRTI backbone of tenofovir disoproxil fumarate (TDF) and lamivudine (3TC). They were randomized to an efavirenz (EFV) arm (600 mg oncedaily), a RAL arm (400 mg twice-daily), or a high dose RAL $\operatorname{arm}(800 \mathrm{mg}$ twice-daily). Proportions of subjects achieving the primary endpoint of a HIV RNA $<50$ copies $/ \mathrm{mL}$ at 48 weeks were 73\% (EFV group), 75\% (RAL $400 \mathrm{mg}$ group), and $65 \%$ (RAL $800 \mathrm{mg}$ group). Both RAL groups exhibited good safety profiles. Therefore, despite previous PK alterations, RAL dosed at $400 \mathrm{mg}$ twice-daily appears to be a suitable alternative to EFV in adult patients cotreated with RIF. ${ }^{51}$ 
Until regulatory authorities such as the FDA revise dosing recommendations for adults during RIF cotreatment, it is advisable to continue to double the dose of RAL during RIF cotreatment. A recent short report suggested that during RIFcotreatment, RAL tablets administered by chewing, rather than swallowing whole tablets, may improve the overall PK profile. This strategy requires further evaluation. ${ }^{52}$

The effects of rifabutin and rifapentine on the PK profile of RAL have also been studied in adult patients. Administration of either daily rifabutin (used in the treatment of active TB) or weekly rifapentine in combination with isoniazid (used for the management of latent Mycobacterium tuberculosis infection) did not significantly alter the PK profile of RAL. ${ }^{53,54}$ Similar studies - particularly those exploring the interaction between RIF and RAL - have not yet been completed in children and adolescents.

\section{ARV agents}

Although not extensively studied, RAL does not appear to interact in a clinically significant manner with NRTIs, including 3TC, abacavir (ABC), and TDF. ${ }^{55-58} \mathrm{PK}$ studies suggest that when RAL is coadministered with EFV, an NNRTI dose adjustment is not required. ${ }^{59}$ Etravirine (ETR), an inducer of UGT isoenzyme 1A1, exerts a modest depressive effect on the PK profile of RAL; this effect is not considered to be of clinical significance. ${ }^{60,61}$

The interaction between RAL and the PIs that are commonly used in pediatric practice has been explored in adult studies. Although ritonavir (RTV) is an inducer of glucuronosyltransferases, when administered in a standard dose as a boosting agent it does not significantly alter the PK profile of RAL. ${ }^{59}$ The coadministration of lopinavir/ritonavir (LPV/ RTV) coformulated tablets and RAL at standard adult dosages resulted in a $30 \%$ reduction in the $\mathrm{RAL} \mathrm{C}_{\text {trough }}$. Dose adjustments were not required because sufficient exposure was maintained relative to the $\mathrm{IC}_{95}{ }^{6}{ }^{62}$ The administration of additional RTV to young HIV-infected children - via a LPV/RTV-containing regimen during treatment for TB coinfection - is employed to overcome the effect of RIF on the metabolism of LPV. ${ }^{63}$ The consequence of this super-boosting strategy on RAL PK has not been evaluated.

Atazanavir (ATV) in combination with RTV is increasingly favored as the preferred PI for older children because of its advantageous toxicity profile. Several studies have shown that ATV with or without the addition of RTV results in a moderate increase in RAL exposure. However, dose adjustments are not considered necessary. ${ }^{18,64-67}$ Darunavir (DRV) boosted by RTV is a consideration in pediatric salvage regimens. Studies have documented either no change or a modest decrease in plasma and cellular RAL concentrations when administered DRV in combination with RTV. ${ }^{68,69}$

\section{Other drug interactions}

There has been limited research on the interaction of RAL with other drugs that are commonly used in pediatric HIV practice. For example, the potential impact of anticonvulsants such as phenytoin, phenobarbitone, valproic acid, and lamotrigine on the PK profile of RAL has not been adequately investigated. ${ }^{18}$ Phenytoin induces the activity of uridine glucuronyl transferases and valproic acid inhibits both cytochrome P450 enzymes and uridine glucuronyl transferases, but whether they significantly alter RAL exposure has not been specifically researched. ${ }^{70,71}$ Lamotrigine does not alter the activity of UGT isoenzyme 1A, suggesting that RAL dose-adjustment should not be required during coadministration. ${ }^{70}$ Studies have shown that RAL does not alter the exposure of lamotrigine or midazolam during coadministration. ${ }^{71-74}$ Ongoing research should provide a more detailed understanding of drug interactions involving RAL, particularly in the pediatric setting where there is currently very little research. For the present time, the US FDA has a cautionary on the use of RAL in combination with other drugs which are strong inducers of UGT isoenzyme 1A1 especially where the effects of these agents on RAL metabolism have not been adequately evaluated.

\section{Efficacy \\ Adult studies}

Numerous studies in adult patients have demonstrated the potent ARV activity of RAL against HIV-1 in both ARV-naïve and ARV-experienced patients. . $^{23,24,75,76}$

Gotuzzo et $\mathrm{al}^{23}$ demonstrated safety and efficacy of RAL in comparison to EFV in combination with TDF/3TC; the 96-week data of the STARTMRK trial led to US FDA approval of RAL in ARV-naïve patients. ${ }^{24,76}$ In this latter noninferiority study, RAL was compared to EFV in combination with TDF/emtricitabine (FTC) in 566 ARV-naïve patients. The RAL arm achieved a shorter time to viral suppression than the EFV arm, and non-inferiority was demonstrated at 48 weeks and maintained to 156 weeks. At 156 weeks, a significantly higher number of study subjects in the RAL arm had achieved HIV-1 RNA $<50$ copies/mL than in the EFV arm $(75.4 \%$ versus $68.1 \%)$ and the mean CD4+ cell count increase was significantly greater in the RAL arm. ${ }^{76}$ The QDMRK trial confirmed the efficacy of RAL $400 \mathrm{mg}$ twice- 
daily with TDF/FTC in ARV-naïve patients. ${ }^{23}$ As a result of these studies, RAL was included as one of the preferred agents for ARV-naïve adults in the 2011 Department of Health and Human Services (DHHS) and the 2010 International AIDS Society (IAS) guidelines. ${ }^{77,78}$ Twice-daily dosing of RAL and cost considerations make RAL most beneficial in situations where NNRTI- or PI-based first-line ARV regimens are less favorable. ${ }^{79}$

Adult studies investigating nucleoside/nucleotide-sparing first-line regimens in order to avoid NRTI-associated toxicities have focused on RAL in combination with a PI (DRV or LPV with RTV boosting, or ATV with or without RTV boosting). Although efficacy outcomes in non-comparative and comparative trials are similar to RAL in combination with dual NRTI regimens, NRTI-sparing regimens containing RAL have been associated with increased rates of RAL resistance and are not currently recommended for ARV-naïve patients able to take other regimens. ${ }^{79-85}$

In ARV-experienced patients without previous exposure to RAL, there is currently little risk of primary resistance to the integrase inhibitor class of drugs. ${ }^{86}$ The addition of RAL to OBTs has shown improved virological responses compared to OBTs alone. ${ }^{87-93}$ RAL compared to placebo given in combination with OBTs in the BENCHMRK 1 and 2 trials resulted in an undetectable viral load after 96 weeks in $56 \%(266 / 466)$ versus $26 \%(62 / 237)$ of study subjects in the RAL and placebo treated arms, respectively. ${ }^{87}$ The RAL arm also had significantly greater decreases in HIV-1 RNA and increases in CD4+ cell counts than the placebo arm. These findings were the basis for the US FDA approval of RAL for ARV-experienced adults. ${ }^{87}$ For highly treatment-experienced patients who lack active ARV options in their OBT, RAL may still show virologic efficacy but the risk of RAL resistance is increased as a result of functional monotherapy. RAL resistance results from mutations within the integrase protein coding sequence and has been documented on resistance assays as early as 1 month after treatment initiation in RAL-naïve patients. ${ }^{94-96}$ Patients with mutations involving the two principal resistance pathways (N155 and Q148) have shown no residual ARV activity when continuing RAL treatment. ${ }^{43}$

A number of switch studies have investigated the utility of RAL as an alternative agent in stable virologically suppressed patients on ART. Switching from enfuvirtide (ENF) - an HIV-1 fusion inhibitor administered twice-daily by subcutaneous injection and associated with frequent injection site reactions - to RAL without changing OBT is associated with safety, virologic efficacy, and improved treatment tolerability. ${ }^{89,93}$ Conflicting results have been reported with switching from RTV-boosted PI regimens to RAL-based regimens in order to simplify treatment and reduce potential adverse effects. In two Phase III randomized, controlled trials (SWITCHMRK 1 and 2), switching to RAL from LPV/RTV in patients virologically suppressed $(<50$ copies $/ \mathrm{mL}$ for at least 3 months) showed reductions in lipid concentrations but a less than expected virologic efficacy in the RAL arm; the study was stopped prematurely. ${ }^{90}$ The SPIRAL study evaluated switching from any RTV-boosted PI regimen to RAL in patients virologically suppressed for the previous 6 months; it demonstrated the virologic non-inferiority of switching to RAL. ${ }^{91}$ This study highlighted that longer duration of virologic suppression is associated with reduced risk of virologic failure after switching; it also emphasizes the importance of assessing past resistance and of maintaining fully active OBT prior to considering switching from a boosted PI to a RAL regimen.

\section{Pediatric studies}

Preliminary results for IMPAACT P1066 study cohorts I and II (children 6-18 years) were presented in $2009^{43}$ and suggested that RAL plus OBT was effective in these treatment-experienced children; viral suppression rates of $80 \%(<400$ copies $/ \mathrm{mL})$ and $63 \%(<50$ copies $/ \mathrm{mL})$ at 12 weeks were achieved and early immunological responses were favorable.

Study cohorts I-III (children 2-18 years) have been completed and reported acceptable efficacy in children 6-18 years receiving the adult tablet, and children 6-12 years and 2-5 years receiving the pediatric chewable tablets. ${ }^{39,41}$ Among 96 HIV-1 infected treatment-experienced children aged 2-18 years treated with RAL and OBT for 48 weeks, $>75 \%$ achieved $>1 \log 10$ HIV RNA decline or $<400$ copies/mL, and $>50 \%$ achieved HIV RNA $<50$ copies/ $\mathrm{mL},{ }^{45}$ Although safety results were reported, other clinical efficacy end-points were not described. ${ }^{39,41}$ Recommended age and weight-based doses of adult and pediatric tablets in these age groups have been established; US FDA approval was obtained in December 2011. ${ }^{11}$

Study cohorts IV and V (children 4 weeks-2 years) evaluating RAL oral granule formulation are ongoing. Nine children in cohort IV, either ARV-experienced or having failed PMTCT, with a mean age of 13 months and mean $\log _{10}$ HIV RNA and CD4+ count/percentage of 5.68 and 1338 cells/ $\mu \mathrm{L} / 21.4 \%$, respectively, have been enrolled. After 24 weeks on a RAL-containing regimen, $>80 \%$ of children $(n=7)$ achieved $\geq 1 \log _{10}$ HIV RNA decline from baseline or $<400$ copies/mL, and the median increase in CD4+ count/ percentage was 446 cells $/ \mathrm{mm}^{3} / 5.3 \%{ }^{42} \mathrm{RAL}$ oral granules for suspension are not yet US FDA-approved. 
A retrospective analysis from the French Expanded Access Program ${ }^{97}$ reported on the outcome of 23 HIV-1infected adolescents (17 male, 6 female; median age 15.5 years [range 12-17]; and a median duration of previous ARV therapy 13 years [range 10-17 years]) with multidrugresistant HIV-1 who were treated with RAL in combination with two to five other ARVs selected by the clinician; ETR was used in 16 patients, DRV in 17 patients, and both in 13 . Twenty-one of the patients were treated with RAL because of active viral replication (median HIV-1 RNA level of five $\log _{10}$ copies $/ \mathrm{mL}$ ). Two patients with viral control on treatment, including ENF, were switched to RAL because of injection-site reactions. Median baseline CD4+ cell count prior to RAL treatment was 194 cells/mL (range 5-484) and 22 of 23 had genotype-documented triple-class oral ARV drug resistance. ${ }^{44}$

Follow-up data on 22 of 23 patients with a median treatment duration of 12 months (range 9-21 months) is available. The mean standard deviation (SD) score for weight increased significantly from $-0.82 \mathrm{SD}(-2.63$ to +1.4$)$ to $-0.46 \mathrm{SD}(-2.9$ to +1.8$)(P=0.006)$. No deaths or AIDS-defining clinical events occurred. One patient stopped RAL after 3 weeks due to headache and another stopped after 9 months due to virologic failure.

In 20 patients treated for active viral replication, there was a significant decrease in HIV-1 RNA from mean (SD) of $5.02(0.59)$ at the start of RAL, to $1.74(0.96) \log _{10}$ copies $/ \mathrm{mL}$ at month $9(P<0.001)$. There was a trend towards significance in the difference in the viral load decrease between the 12 patients receiving RAL, ETR, and DRV versus the eight patients receiving other regimens. At the last followup (9-21 months), 21 patients remained on RAL-containing treatment, 18 had viral load $<400$ copies $/ \mathrm{mL}$, and 13 had viral load $<50$ copies/mL. Virologic failure (viral load $>400$ copies/mL at two consecutive points after initial response) occurred in four patients, and was clearly related to suboptimal adherence in two. ${ }^{44}$

In a similar retrospective analysis from Spain, 19 multidrug-resistant children and adolescents (median age 16 years, median baseline HIV-1 RNA $4 \log _{10}$ copies/mL, and median CD4+ cell count 329) were treated with RAL-containing salvage ART (OBT contained at least one fully active drug in $17 / 19$ [89\%] of patients). Patients were followed-up for a median of 80 weeks (interquartile range [IQR] 49.4-96.4 weeks). ARV regimens included DRV/RTV in nine (47\%) patients and combination of DRV/RTV and ETR in six (32\%). Maraviroc was administered to four (21\%) patients and ENF to another four (21\%). After starting RAL-containing ART,
17/19 (89.5\%) and 13/19 (68.4\%) achieved HIV-1 RNA levels of $<400$ and $<50$ copies $/ \mathrm{mL}$, respectively, and the median CD4+ cell count was 636 (IQR 358-879) cells $/ \mathrm{mm}^{3}$. No deaths or AIDS-defining illnesses were recorded. None of the patients with virologic non-suppression developed resistance mutations to RAL.

\section{Safety and tolerability Adult data}

Among healthy male adult volunteers who were exposed to RAL for 10 days, headache and fatigue were most commonly reported, all transient and mild or moderate in intensity. No dose-response relationship was reported, even at the highest dose of $1,600 \mathrm{mg} /$ day. No laboratory abnormalities or disorders of cardiac conduction were identified. ${ }^{17}$

In HIV-infected adult subjects, RAL was well tolerated compared to EFV and comparator PIs in Phase II and III studies. Mild and transient headache, other nervous system, and gastrointestinal events were the most common effects reported..$^{98}$ In the BENCHMRK studies, nervous system events (mainly headache) were noted at a similar incidence to placebo. In the STARTMRK study, neuropsychiatric symptoms were significantly lower compared directly with EFV (26\% versus 59\%, $P<0.0001$ at 48 weeks). ${ }^{88,99}$

Skin hypersensitivity reactions were uncommon and mild, comparable with placebo (5.3\% versus $2.5 \%)$, and confounded by other drugs. Rare cases of Stevens-Johnson syndrome were reported post-marketing, although true causality has been difficult to establish. ${ }^{18}$

RAL has a neutral effect on lipid metabolism. ${ }^{100,101}$ Compared with EFV, RAL demonstrated statistically significant favorable changes in plasma lipid and fasting blood glucose concentrations in a previously treatment-naïve population. The effect on body composition was similar, however, with truncal and appendicular fat gain comparable in both groups. Lipoatrophy was uncommon $(8 \%$ versus $5 \%$, respectively) in both groups. ${ }^{76}$ No change in body composition was found in a group 48 weeks after switching to RAL, whereas in the group maintaining PI therapy total and visceral fat increased. Bone mineral density increased in the RAL switch group, but it was unchanged in the PI maintenance group. ${ }^{45}$

In the BENCHMRK studies, a higher incidence of malignancies was observed in the RAL treated arm $(3.5 \%$ versus $1.7 \%$ in placebo-treated arm) at 48 weeks, a finding highlighted in the US FDA safety analysis. No pattern was evident in the number or type of malignancies, and no trend in the time of onset. At 96 weeks, with adjustment for 
person-years of follow-up during the double-blind phases, the development of cancer was comparable between the RAL and placebo arms. ${ }^{88,98}$

Pooled Phase II and III data from a US FDA safety analysis indicate a serum creatine kinase elevation of all grades in RAL-treated arms (13.9\% versus 9.4\%). ${ }^{98}$ This difference persisted ( $4.2 \%$ versus $2.5 \%)$ when stratified to elevations of grade $3 / 4$, and also when corrected for concomitant use of statins or fenofibrate. However, no clinical musculoskeletal effects were reported and no participants were withdrawn from the study due to creatine kinase elevation. Nevertheless, the US FDA issued a cautionary note on the use of RAL in patients with existing myopathy, as prospective cohort studies have demonstrated grade $3 / 4$ creatine kinase elevation of between $5 \%$ and $13 \% .^{98}$

\section{Pediatric data}

Cumulative safety data for the IMPAACT P1066 cohorts of patients 2-18 years of age were presented at the 19th International AIDS conference in $2012 .{ }^{45}$ Ninety-six ARVexperienced subjects were treated with RAL (400 mg twice-daily of film-coated tablet [6-18 years] and weightbased dosing [approximately $6 \mathrm{mg} / \mathrm{kg}$ twice-daily] of RAL chewable tablet [2 to $<12$ years]) in combination with OBT. By week 48, 15 subjects had experienced Grade 3+ clinical adverse events (AE) (one subject with drug related [DR] psychomotor hyperactivity, abnormal behavior, and insomnia) and 16 subjects developed Grade 3+ laboratory AEs (one with DR aspartate transaminase [AST] and alanine transaminase [ALT] elevations). Fifteen subjects had serious clinical AEs (one with DR rash) and two subjects developed serious laboratory AEs (one with DR transaminase increase). There were no discontinuations due to AEs and no deaths. ${ }^{45}$

RAL was generally well tolerated in nine subjects enrolled in IMPAACT P1066 cohort IV ( 6 months to $<2$ years of age) receiving RAL granules for suspension by weight-based dosing. However, three subjects accounted for 16 grade $\geq 3$ adverse events, two of which were considered related to RAL (elevated bilirubin and hypoglycemia). ${ }^{42}$

Among 23 adolescents with multidrug-resistant HIV-1 who were treated with RAL in combination with OBT in the French Expanded Access Program, one patient stopped treatment after 3 weeks due to headache, but no other moderateto-severe clinical side-effects. No grade 3 or 4 laboratory abnormalities occurred. ${ }^{44}$

In a Spanish cohort of 19 multidrug-resistant children and adolescents who were treated with RAL-containing salvage ART and followed-up for a median of 80 weeks, no symptoms of severe intolerance were recorded. Two patients experienced mild-moderate short-term skin rash.

\section{Conclusion}

RAL, currently commercially available as film-coated tablets (400 mg) and chewable tablets (100 mg and $25 \mathrm{mg}$ ), is US FDA-approved for treatment of HIV-1 infected children aged $\geq 2$ years and weight $\geq 10 \mathrm{~kg} .{ }^{11}$ Due to insufficient pediatric PK and a lack of safety and efficacy data, integrase inhibitors are not currently recommended as initial therapy in children. ${ }^{2}$

Although many HIV-1 infected individuals initiate empirical first-line ARV regimens, current US guidelines recommend ARV drug-resistance testing with a genotypic assay before initiation of therapy for all treatment-naïve individuals. Use of resistance testing results is also recommended for selection of initial drug combinations. ${ }^{2}$

RAL may be considered for use in ARV-experienced children and adolescents with treatment failure and virologic resistance. Potential roles for RAL are twofold. First, as an alternative to a PI in an ARV regimen with at least two fully active agents after failing a first-line regimen comprising an NNRTI plus two NRTIs. Secondly, it may be used as an alternative to an NNRTI in an ARV regimen with at least two fully active agents after failing a first-line regimen comprising a PI plus two NRTIs. ${ }^{2}$

In ARV-experienced patients with multi-drug resistance and virologic treatment failure requiring a switch to a new ARV regimen, the availability of RAL (as representative of a new ARV drug class to which there is currently widespread HIV susceptibility) increases the likelihood of viral success. In highly treatment-experienced children and adolescents, successful treatment outcomes have been reported using RAL in combination with a new generation PI (usually DRV/RTV) and/or NNRTI (usually ETR) and OBT. ${ }^{44}$

Advantages of RAL relevant to pediatrics include an overall favorable adverse effect profile, availability of chewable tablets (and granules for suspension are being developed for infants and younger children), and that ingestion can occur with food.

Potential disadvantages of current RAL use in children include the twice-daily dosing schedule and the lack of PK, safety, and effectiveness data on RAL-containing ART in children receiving anti-TB medication. The forthcoming IMPAACT P1101 study will evaluate this latter issue. Lack of pediatric data on potential drug interactions between 
RAL and ATV/RTV, DRV/RTV, super-boosted LPV/RTV, and many other drugs, currently limits more widespread use of RAL. In addition, in many resource-limited settings where new ARV options are urgently required, RAL is not yet available or affordable.

Newer integrase inhibitors include elvitegravir, which requires boosting with cobicistat or RTV but is administered once-daily, and dolutegravir, active in patients with RAL-resistance mutations. ${ }^{79}$ Elvitegravir is currently only available as a fixed-dose combination tablet containing elvitegravir/cobicistat/FTC/TDF, and is US FDA-approved for use as a complete ARV regimen in HIV-1-infected ARV treatment-naïve adults. There are no data yet on its use in children aged $<18$ years, although a pediatric dose-finding study of dolutegravir is underway through the IMPAACT network. $^{2}$

The potential role of RAL as part of combination ART in TB coinfected children taking RIF-containing TB medication, as well as in the context of PMTCT both for pregnant HIV-infected woman and for HIV-exposed infant prophylaxis, are being evaluated. The integrase inhibitor class of ARV drugs shows great promise in the management of pediatric HIV infection.

\section{Disclosure}

The authors report no conflicts of interest in this work.

\section{References}

1. Violari A, Cotton MF, Gibb DM, et al. Early antiretroviral therapy and mortality among HIV-infected infants. N Engl J Med. 2008; 359(21):2233-2244.

2. Panel on Antiretroviral Therapy and Medical Management of HIVInfected Children. Guidelines for the Use of Antiretroviral Agents in Pediatric HIV Infection. Washington: United States Department of Health and Human Services; 2013. Available at: http://aidsinfo. nih.gov/contentfiles/lvguidelines/pediatricguidelines.pdf. Accessed May 12, 2013.

3. Brady MT, Oleske JM, Williams PL, et al. Declines in mortality rates and changes in causes of death in HIV-1-infected children during the HAART era. J Acquir Immune Defic Syndr. 2010;53(1):86-94.

4. Viani RM, Araneta MR, Deville JG, Spector SA. Decrease in hospitalization and mortality rates among children with perinatally acquired HIV type 1 infection receiving highly active antiretroviral therapy. Clin Infect Dis. 2004;39(5):725-731.

5. Nachman SA, Lindsey JC, Moye J, et al; Pediatric AIDS Clinical Trials Group 377 Study Team. Growth of human immunodeficiency virusinfected children receiving highly active antiretroviral therapy. Pediatr Infect Dis J. 2005;24(4):352-357.

6. Lindsey JC, Malee KM, Brouwers P, Hughes MD; PACTG 219C Study Team. Neurodevelopmental functioning in HIV-infected infants and young children before and after the introduction of protease inhibitor-based highly active antiretroviral therapy. Pediatrics. 2007;119(3):e681-e693.

7. Delaugerre C, Chaix ML, Blanche S, et al; ANRS French Perinatal Cohort. Perinatal acquisition of drug-resistant HIV-1 infection: mechanisms and long-term outcome. Retrovirology. 2009;6:85.
8. Persaud D, Palumbo P, Ziemniak C, et al; Pediatric AIDS Clinical Trials Group P1030 Team. Early archiving and predominance of nonnucleoside reverse transcriptase inhibitor-resistant HIV-1 among recently infected infants born in the United States. J Infect Dis. 2007;195(10):1402-1410.

9. Van Dyke RB, Wang L, Williams PL; Pediatric AIDS Clinical Trials Group 219C Team. Toxicities associated with dual nucleoside reverse transcriptase inhibitor regimens in HIV-infected children. $J$ Infect Dis. 2008;198(11):1599-1608.

10. Foster C, Lyall H. HIV and mitochondrial toxicity in children. J Antimicrob Chemother. 2008;61(1):8-12.

11. Isentress ${ }^{\circledR}$ (raltegravir) [product information]. Whitehouse Station, NJ: Merck and Co, Inc; 2011.

12. Cherepanov P, Maertens GN, Hare S. Structural insights into the retroviral DNA integration apparatus. Curr Opin Struct Biol. 2011; 21(2):249-256.

13. Krishnan L, Engelman A. Retroviral integrase proteins and HIV-1 DNA integration. J Biol Chem. 2012;287(49):40858-40866.

14. Craigie R, Bushman FD. HIV DNA Integration. Cold Spring Harb Perspect Med. 2012;2(7):a006890.

15. Malet I, Calvez V, Marcelin AG. The future of integrase inhibitors of HIV-1. Curr Opin Virol. 2012;2(5):580-587.

16. Hazuda DJ, Felock P, Witmer M, et al. Inhibitors of strand transfer that prevent integration and inhibit HIV-1 replication in cells. Science. 2000;287(5453):646-650.

17. Iwamoto M, Wenning LA, Petry AS, et al. Safety, tolerability, and pharmacokinetics of raltegravir after single and multiple doses in healthy subjects. Clin Pharmacol Ther. 2008;83(2):293-299.

18. Isentress (raltegravir) [prescribing information]. Whitehouse Station, NJ: Merck and Co, Inc; 2012.

19. Markowitz M, Morales-Ramirez JO, Nguyen BY, et al. Antiretroviral activity, pharmacokinetics, and tolerability of MK-0518, a novel inhibitor of HIV-1 integrase, dosed as monotherapy for 10 days in treatment-naive HIV-1-infected individuals. $J$ Acquir Immune Defic Syndr. 2006;43(5):509-515.

20. Ter Heine R, Mulder JW, van Gorp EC, Wagenaar JF, Beijnen JH, Huitema AD. Intracellular and plasma steady-state pharmacokinetics of raltegravir, darunavir, etravirine and ritonavir in heavily pre-treated HIV-infected patients. Br J Clin Pharmacol. 2010;69(5):475-483.

21. Adams JL, Greener BN, Kashuba AD. Pharmacology of HIV integrase inhibitors. Curr Opin HIV AIDS. 2012;7(5):390-400.

22. Eron JJ, Rockstroh JK, Reynes J, et al; QDMRK Investigators. Raltegravir once daily or twice daily in previously untreated patients with HIV-1: a randomised, active-controlled, phase 3 non-inferiority trial. Lancet Infect Dis. 2011;11(12):907-915.

23. Gotuzzo E, Nguyen B, Markowitz M, et al. Sustained efficacy and tolerability of raltegravir after 240 weeks of combination ART in treatment-naïve HIV-1 infected patients; final analysis of protocol 004. Presented at: The 6th International AIDS Society; Rome, Italy; July 17-20, 2011. Abstract WEPDB0102.

24. Rockstroh JK, Lennox JL, Dejesus E, et al; STARTMRK Investigators. Long-term treatment with raltegravir or efavirenz combined with tenofovir/emtricitabine for treatment-naive human immunodeficiency virus-1-infected patients: 156-week results from STARTMRK. Clin Infect Dis. 2011;53(8):807-816.

25. Rizk ML, Hang Y, Luo WL, et al. Pharmacokinetics and pharmacodynamics of once-daily versus twice-daily raltegravir in treatmentnaive HIV-infected patients. Antimicrob Agents Chemother. 2012; 56(6):3101-3106.

26. Yilmaz A, Gisslén M, Spudich S, et al. Raltegravir cerebrospinal fluid concentrations in HIV-1 infection. PLoS ONE. 2009;4(9):e6877.

27. Croteau D, Letendre S, Best BM, et al; CHARTER Group. Total raltegravir concentrations in cerebrospinal fluid exceed the 50-percent inhibitory concentration for wild-type HIV-1. Antimicrob Agents Chemother. 2010;54(12):5156-5160.

28. Mora-Peris B, Mackie NE, Suan D, Cooper DA, Brew BJ, Winston A. Raltegravir resistance in the cerebrospinal fluid. Infection. 2013;41(3): 731-734. 
29. Siccardi M, D’ Avolio A, Rodriguez-Novoa S, et al. Intrapatient and interpatient pharmacokinetic variability of raltegravir in the clinical setting. Ther Drug Monit. 2012;34(2):232-235.

30. Cattaneo D, Gervasoni C, Meraviglia P, et al. Inter- and intra-patient variability of raltegravir pharmacokinetics in HIV-1-infected subjects. J Antimicrob Chemother. 2012;67(2):460-464.

31. Cattaneo D, Baldelli S, Cerea M, et al. Comparison of the in vivo pharmacokinetics and in vitro dissolution of raltegravir in HIV patients receiving the drug by swallowing or by chewing. Antimicrob Agents Chemother. 2012;56(12):6132-6136.

32. Brainard DM, Friedman EJ, Jin B, et al. Effect of low-, moderate-, and high-fat meals on raltegravir pharmacokinetics. J Clin Pharmacol. 2011;51(3):422-427.

33. Kassahun K, McIntosh I, Cui D, et al. Metabolism and disposition in humans of raltegravir (MK-0518), an anti-AIDS drug targeting the human immunodeficiency virus 1 integrase enzyme. Drug Metab Dispos. 2007;35(9):1657-1663.

34. Wenning LA, Petry AS, Kost JT, et al. Pharmacokinetics of raltegravir in individuals with UGT1A1 polymorphisms. Clin Pharmacol Ther. 2009;85(6):623-627.

35. Iwamoto M, Hanley WD, Petry AS, et al. Lack of a clinically important effect of moderate hepatic insufficiency and severe renal insufficiency on raltegravir pharmacokinetics. Antimicrob Agents Chemother. 2009;53(5):1747-1752.

36. Barau C, Braun J, Vincent C, et al; ANRS 148 Study Group. Pharmacokinetic Study of Raltegravir in $\mathrm{HIV}^{+}$Patients with End Stage Liver Disease: LIVERAL ANRS 148 Study. Presented at: 20th Conference on Retroviruses and Opportunistic Infections; Atlanta, GA; March 3-6, 2013. Paper \#528.

37. Acosta A, Wiznia A, Nachman S, et al. Raltegravir (RAL) pharmacokinetics (PK) in adolescents: preliminary results from IMPAACT protocol 1066. Presented at: 9th International Workshop on Clinical Pharmacology of HIV Therapy; New Orleans, LA; April 7-9, 2008.

38. Nachman S, Acosta E, Wiznia A, et al. Raltegravir phamacokinetics (PK) and safety in adolescents: Preliminary results from IMPAACT P1066. Presented at: 48th Interscience Conference on Antimicrobial Agents and Chemotherapy (ICAAC); October 25-28, 2008; Washington, DC.

39. Nachman S, Samson P, Acosta E, et al. P1066 Group. Pharmacokinetic (PK), Safety, and Efficacy Data on Cohort IIA; Youth Aged 6 to 11 Years from IMPAACT P1066: A Phase I/II Study to Evaluate Raltegravir (RAL) in HIV-1 Infected Youth. Presented at: 17th Conference on Retroviruses and Opportunistic Infections; San Francisco, CA; February 16-19, 2010. Paper \#873.

40. Nachman S, Acosta E, Samson P, et al. P1066 Group. Interim Results from IMPAACT P1066: Raltegravir (RAL) Oral Chewable Tablet (OCT) Formulation in Children 6 to 11 Years. Presented at: 17th Conference on Retroviruses and Opportunistic Infections; San Francisco, CA; February 16-19, 2010. Paper \#161LB.

41. Nachman S, Acosta E, Zheng N, et al; P1066 Study Team. Interim Results from IMPAACT P1066: RAL Oral Chewable Tablet Formulation for 2- to 5-Year-olds. Presented at: 18th Conference on Retroviruses and Opportunistic Infections; Boston, MA; February 27-March 2, 2011. Paper \#715.

42. Spector S, Acosta E, Zheng N, et al; IMPAACT P1066 Study Team. Raltegravir Oral Granules Formulation in Children 6 months to $<2$ Years of Age: Interim Results from IMPAACT P1066. Presented at: 19th Conference on Retroviruses and Opportunistic Infections; Seattle, WA; March 5-8, 2012. Paper \#987.

43. Wiznia A, Samson P, Acosta E, et al. Safety and efficacy of raltegravir in pediatric HIV infection. Preliminary analysis from the international maternal pediatric adolescent AIDS clinical trials group, P1066. Abstract presented at: 16th Conference on Retroviruses and Opportunistic Infections. Montreal, Quebec; February 8-11, 2009. Abstract 874.

44. Briz V, León-Leal JA, Palladino C, et al. Potent and sustained antiviral response of raltegravir-based highly active antiretroviral therapy in HIV type 1-infected children and adolescents. Pediatr Infect Dis J. 2012;31(3):273-277.
45. Nachman S, Acosta E, Zheng N, et al. IMPAACT P1066: raltegravir (RAL) safety and efficacy in HIV infected (+) youth two to 18 years of age through week 48. Presented at: XIX International AIDS Conference; July 22-27, 2012; Washington, DC. Abstract TUAB0205.

46. Brainard DM, Gendrano III N, Jin B, et al. A Pharmacokinetic Comparison of Adult and Pediatric Formulations of RAL in Healthy Adults. Presented at: 17th Conference on Retroviruses and Opportunistic Infections; San Francisco, CA; February 16-19, 2010. Paper \#872.

47. McKeown DA, Rosenvinge M, Donaghy S, et al. High neonatal concentrations of raltegravir following transplacental transfer in HIV-1 positive pregnant women. AIDS. 2010;24(15):2416-2418.

48. Hegazi A, Mc Keown D, Doerholt K, Donaghy S, Sadiq ST, Hay P. Raltegravir in the prevention of mother-to-child transmission of HIV-1: effective transplacental transfer and delayed plasma clearance observed in preterm neonates. AIDS. 2012;26(18):2421-2423.

49. Clarke D, Acosta E, Rizk M, et al; IMPAACT P1097 Study Team. Raltegravir Pharmacokinetics and Safety in Neonates (IMPAACT P1097). Presented at: 20th Conference on Retroviruses and Opportunistic Infections; Atlanta, GA; March 3-6, 2013. Paper \#974.

50. Wenning LA, Hanley WD, Brainard DM, et al. Effect of rifampin, a potent inducer of drug-metabolizing enzymes, on the pharmacokinetics of raltegravir. Antimicrob Agents Chemother. 2009;53(7): 2852-2856.

51. Grinsztejn B, De Castro N, Arnold V, et al. Efficacy and Safety of Raltegravir vs Efavirenz for the Treatment of HIV/TB Patients: 48-Week Results of the ANRS 12180 Reflate TB Trial. Presented at: 20th Conference on Retroviruses and Opportunistic Infections; Atlanta, GA; March 3-6, 2013. Paper \#853.

52. Gervasoni C, Riva A, Impagnatiello C, Galli M, Cattaneo D. Is Chewed Raltegravir an Option to Care for HIV-Infected Patients With Active Tuberculosis? Clin Infect Dis. 2013. Epub May 10, 2013. Brainard DM, Kassahun K, Wenning LA, et al. Lack of a clinically meaningful pharmacokinetic effect of rifabutin on raltegravir: in vitro/in vivo correlation. J Clin Pharmacol. 2011;51(6):943-950.

53. Weiner M, Peloquin C, Engle M, Egelund E, Thomas P, Mac Kenzie W. The pharmacokinetic interaction between raltegravir and rifapentine in healthy volunteers. Presented at: 19th Conference on Retroviruses and Opportunistic Infections; Seattle, WA; March 5-8, 2012. Paper 615.

54. Nguyen BY, Isaacs RD, Teppler H, et al. Raltegravir: the first HIV-1 integrase strand transfer inhibitor in the HIV armamentarium. Ann NY Acad Sci. 2011;1222:83-89.

55. Jackson A, Moyle G, Dickinson L, et al. Pharmacokinetics of abacavir and its anabolite carbovir triphosphate without and with darunavir/ ritonavir or raltegravir in HIV-infected subjects. Antivir Ther (Lond). 2012;17(1):19-24.

56. Brainard DM, Wenning LA, Stone JA, Wagner JA, Iwamoto M. Clinical pharmacology profile of raltegravir, an HIV-1 integrase strand transfer inhibitor. J Clin Pharmacol. 2011;51(10):1376-1402.

57. Wenning LA, Friedman EJ, Kost JT, et al. Lack of a significant drug interaction between raltegravir and tenofovir. Antimicrob Agents Chemother. 2008;52(9):3253-3258.

58. Iwamoto M, Wenning LA, Petry AS, et al. Minimal effects of ritonavir and efavirenz on the pharmacokinetics of raltegravir. Antimicrob Agents Chemother. 2008;52(12):4338-4343.

59. Anderson MS, Kakuda TN, Hanley W, et al. Minimal pharmacokinetic interaction between the human immunodeficiency virus nonnucleoside reverse transcriptase inhibitor etravirine and the integrase inhibitor raltegravir in healthy subjects. Antimicrob Agents Chemother. 2008;52(12):4228-4232.

60. Do VT, Higginson RT, Fulco PP. Raltegravir dosage adjustment in HIV-infected patients receiving etravirine. Am J Health Syst Pharm. 2011;68(21):2049-2054.

61. Rhame F, Long M, Acosta E. RAL-KAL: pharmacokinetics (PK) of coadministered raltegravir (RAL) and lopinavir-ritonavir (KAL) in healthy adults. Presented at: AIDS 2008 - XVII International AIDS Conference; Mexico City, Mexico; August 3-8, 2008. Abstract TUPE0075. 
62. Ren Y, Nuttall JJ, Egbers C, et al. Effect of rifampicin on lopinavir pharmacokinetics in HIV-infected children with tuberculosis. J Acquir Immune Defic Syndr. 2008;47(5):566-569.

63. Iwamoto M, Wenning LA, Mistry GC, et al. uridine diphosphate glucuronosyltransferase isoenzyme 1A modestly increases plasma levels of raltegravir in healthy subjects. Clin Infect Dis. 2008;47(1):137-140.

64. Zhu L, Butterton J, Persson A, et al. Pharmacokinetics and safety of twice-daily atazanavir $300 \mathrm{mg}$ and raltegravir $400 \mathrm{mg}$ in healthy individuals. Antivir Ther (Lond). 2010;15(8):1107-1114.

65. Cattaneo D, Ripamonti D, Baldelli S, Cozzi V, Conti F, Clementi E. Exposure-related effects of atazanavir on the pharmacokinetics of raltegravir in HIV-1-infected patients. Ther Drug Monit. 2010;32(6): 782-786.

66. Neely M, Decosterd L, Fayet A, et al. Pharmacokinetics and pharmacogenomics of once-daily raltegravir and atazanavir in healthy volunteers. Antimicrob Agents Chemother. 2010;54(11): 4619-4625.

67. Fayet Mello A, Buclin T, Franc C, et al. Cell disposition of raltegravir and newer antiretrovirals in HIV-infected patients: high inter-individual variability in raltegravir cellular penetration. JAntimicrob Chemother. 2011;66(7):1573-1581.

68. Jackson A, Watson V, Back D, et al. Plasma and intracellular pharmacokinetics of darunavir/ritonavir once daily and raltegravir once and twice daily in HIV-infected individuals. J Acquir Immune Defic Syndr. 2011;58(5):450-457.

69. Johannessen SI, Landmark CJ. Antiepileptic drug interactions principles and clinical implications. Curr Neuropharmacol. 2010;8(3): 254-267.

70. DiCenzo R, Peterson D, Cruttenden K, et al. Effects of valproic acid coadministration on plasma efavirenz and lopinavir concentrations in human immunodeficiency virus-infected adults. Antimicrob Agents Chemother. 2004;48(11):4328-4331.

71. van Luin M, Colbers A, Verwey-van Wissen CP, et al. The effect of raltegravir on the glucuronidation of lamotrigine. J Clin Pharmacol. 2009;49(10):1220-1227.

72. Iwamoto M, Kassahun K, Troyer MD, et al. Lack of a pharmacokinetic effect of raltegravir on midazolam: in vitro/in vivo correlation. $J$ Clin Pharmacol. 2008;48(2):209-214.

73. Birbeck GL, French JA, Perucca E, et al; Quality Standards Subcommittee Of The American Academy Of Neurology; Ad Hoc Task Force Of The Commission On Therapeutic Strategies Of The International League Against Epilepsy. Antiepileptic drug selection for people with HIV/AIDS: evidence-based guidelines from the ILAE and AAN. Epilepsia. 2012;53(1):207-214.

74. Young B, Vanig T, DeJesus E, et al. 96-Week results of a pilot study of abacavir/lamivudine and raltegravir in antiretroviral-naïve HIV-1 infected patients: the SHIELD trial. HIV Clin Trials. 2011;12(4):228-233.

75. Markowitz M, Nguyen BY, Gotuzzo E, et al; Protocol 004 Part II Study Team. Rapid and durable antiretroviral effect of the HIV-1 Integrase inhibitor raltegravir as part of combination therapy in treatment-naive patients with HIV-1 infection: results of a 48-week controlled study. J Acquir Immune Defic Syndr. 2007;46(2):125-133.

76. Department of Health and Human Services. Panel on antiretroviral guidelines for adults and adolescents. Guidelines for the Use of Antiretroviral Agents in HIV-1-Infected Adults and Adolescents. Washington, DC: Department of Health and Human Services; 2011. Available from: http://www.aidsinfo.nih.gov/ContentFiles/AdultandAdolescentGL.pdf. Accessed May 10, 2013.

77. Thompson MA, Aberg JA, Cahn P, et al; International AIDS SocietyUSA. Antiretroviral treatment of adult HIV infection: 2010 recommendations of the International AIDS Society-USA panel. JAMA. 2010;304(3):321-333.

78. Rokas KE, Bookstaver PB, Shamroe CL, et al. Role of raltegravir in HIV-1 management. Ann Pharmacother. 2012;46(4):578-589.

79. Taiwo B, Zheng L, Gallien S, et al; ACTG A5262 Team. Efficacy of a nucleoside-sparing regimen of darunavir/ritonavir plus raltegravir in treatment-naive HIV-1-infected patients (ACTG A5262). AIDS. 2011;25(17):2113-2122.
80. Bedimo R, Drechsler H, Turner D, et al. RADAR study: raltegravir combined with boosted darunavir has similar safety and antiviral efficacy as tenofovir/emtricitabine combined with boosted darunavir in antiretroviral-naïve patients. Presented at: The 6th International AIDS Society; Rome, Italy; July 17-20, 2011. Abstract MOPE214.

81. Reynes J, Lawal A, Pulido F, et al. Examination of noninferiority, safety, and tolerability of lopinavir/ritonavir and raltegravir compared with lopinavir/ ritonavir and tenofovir/emtricitabine in antiretroviral-naïve subjects: the progress study, 48-week results. HIV Clin Trials. 2011;12(5):255-267.

82. Gathe J, Reynes J, Pulido F, et al. Lopinavir/ritonavir (LPV/r) combined with raltegravir (RAL) or tenofovir/emtricitabine (TDF/FTC) in antiretroviral-naïve subjects: 96-week efficacy and safety results of the PROGRESS study. Presented at: The Infectious Disease Society of America; Boston, MA; October 20-23, 2011. Abstract 406

83. Bowman V, Rieg G, Jain S, et al. 48 week results of a pilot randomized study of a nucleoside reverse transcriptase inhibitor (NRTI)-sparing regimen of raltegravir (RAL) + lopinavir/ritonavir (LPV/r) versus efavirenz/tenofovir disoproxil fumarate/emtricitabine (EFV/TDF/FTC) in antiretro. Presented at: The 6th International AIDS Society; Rome, Italy; July 17-20, 2011. Abstract CDB336.

84. Kozal MJ, Lupo S, DeJesus E, et al. The SPARTAN study: a pilot study to assess the safety and efficacy of an investigational NRTI- and RTVsparing regimen of atazanavir (ATV) experimental dose of $300 \mathrm{mg}$ BID plus raltegravir (RAL) $400 \mathrm{mg}$ BID (ATV + RAL) in treatment-naïve HIV-infected subjects. Presented at: The International AIDS Conference; Vienna, Austria; July 18-23, 2010. Abstract THLBB204.

85. Gutiérrez C, Hernández-Novoa B, Pérez-Elías MJ, et al. Prevalence of primary resistance mutations to integrase inhibitors in treatment-naïve and -experienced patients infected with B and non-B HIV-1 variants. HIV Clin Trials. 2013;14(1):10-16.

86. Steigbigel RT, Cooper DA, Kumar PN, et al; BENCHMRK Study Teams. Raltegravir with optimized background therapy for resistant HIV-1 infection. N Engl J Med. 2008;359(4):339-354.

87. Steigbigel RT, Cooper DA, Teppler H, et al; BENCHMRK Study Teamsa. Long-term efficacy and safety of Raltegravir combined with optimized background therapy in treatment-experienced patients with drug-resistant HIV infection: week 96 results of the BENCHMRK 1 and 2 Phase III trials. Clin Infect Dis. 2010;50(4):605-612.

88. Gallien S, Braun J, Delaugerre C, et al; EASIER ANRS 138 Study Group. Efficacy and safety of raltegravir in treatment-experienced HIV1-infected patients switching from enfuvirtide-based regimens: 48 week results of the randomized EASIER ANRS 138 trial. J Antimicrob Chemother. 2011;66(9):2099-2106.

89. Eron JJ, Young B, Cooper DA, et al; SWITCHMRK 1 and 2 investigators. Switch to a raltegravir-based regimen versus continuation of a lopinavirritonavir-based regimen in stable HIV-infected patients with suppressed viraemia (SWITCHMRK 1 and 2): two multicentre, double-blind, randomised controlled trials. Lancet. 2010;375(9712):396-407.

90. Martínez E, Larrousse M, Llibre JM, et al; SPIRAL Study Group. Substitution of raltegravir for ritonavir-boosted protease inhibitors in HIVinfected patients: the SPIRAL study. AIDS. 2010;24(11):1697-1707.

91. Nguyen A, Calmy A, Delhumeau C, et al. A randomized cross-over study to compare raltegravir and efavirenz (SWITCH-ER study). AIDS. 2011;25(12):1481-1487.

92. Towner W, Klein D, Kerrigan HL, Follansbee S, Yu K, Horberg M. Virologic outcomes of changing enfuvirtide to raltegravir in HIV-1 patients well controlled on an enfuvirtide based regimen: 24-week results of the CHEER study. J Acquir Immune Defic Syndr. 2009;51(4):367-373.

93. Métifiot M, Marchand C, Maddali K, PommierY. Resistance to integrase inhibitors. Viruses. 2010;2(7):1347-1366.

94. Fransen S, Gupta S, Danovich R, et al. Loss of raltegravir susceptibility by human immunodeficiency virus type 1 is conferred via multiple nonoverlapping genetic pathways. J Virol. 2009;83(22):11440-11446.

95. Baldanti F, Paolucci S, Gulminetti R, Brandolini M, Barbarini G, Maserati R. Early emergence of raltegravir resistance mutations in patients receiving HAART salvage regimens. J Med Virol. 2010;82(1): $116-122$. 
96. Wirden M, Simon A, Schneider L, et al. Raltegravir has no residual antiviral activity in vivo against HIV-1 with resistance-associated mutations to this drug. J Antimicrob Chemother. 2009;64(5):1087-1090.

97. Thuret I, Tamalet C, Chaix M, et al. Raltegravir in children and adolescents: the French expanded access program. In: 16th Conference on Retroviruses and Opportunistic Infections. Montreal, Quebec; February 8-11, 2009. Abstract 873

98. Lennox JL, DeJesus E, Lazzarin A, et al; STARTMRK investigators. Safety and efficacy of raltegravir-based versus efavirenz-based combination therapy in treatment-naive patients with HIV-1 infection: a multicentre, double-blind randomised controlled trial. Lancet 2009;374(9692):796-806.
99. Pérez-Matute P, Pérez-Martínez L, Blanco JR, Oteo JA. Neutral actions of Raltegravir on adipogenesis, glucose metabolism and lipolysis in 3T3-L1 adipocytes. Curr HIV Res. 2011;9(3):174-179.

100. Minami R, Yamamoto M, Takahama S, Ando H, Miyamura T, Suematsu E. Comparison of the influence of four classes of HIV antiretrovirals on adipogenic differentiation: the minimal effect of raltegravir and atazanavir. J Infect Chemother. 2011;17(2):183-188.

101. Curran A, Martinez E, Saumoy M, et al. Body composition changes after switching from protease inhibitors to raltegravir: SPIRAL-LIP substudy. AIDS. 2012;26(4):475-481.

\section{Publish your work in this journal}

Pediatric Health, Medicine and Therapeutics is an international, peerreviewed, open access journal publishing original research, reports, editorials, reviews and commentaries. All aspects of health maintenance, preventative measures and disease treatment interventions are addressed within the journal. Practitioners from all disciplines are invited to submit their work as well as healthcare researchers and patient support groups. The manuscript management system is completely online and includes a very quick and fair peer-review system. Visit http://www.dovepress.com/ testimonials.php to read real quotes from published authors.

Submit your manuscript here: http://www.dovepress.com/pediatric-health-medicine-and-therapeutics-journal 